\title{
UNA TRADUCCIÓN DEL CELTA HISPANO EN ANTIGUO IRLANDÉS
}

RAMÓN SAINERO

UNED

En la Península Ibérica los estudios sobre las lenguas celtas no han tenido tanta fortuna como en otros países debido eminentemente a la escasez de restos escritos. Gracias a inscripciones sobre metales, monedas y algunas otras fuentes como textos latinos o griegos conocemos algunas palabras y frases del celta que fue hablado en la Península Ibérica. Los textos son bastante reducidos y su traducción problemática. Ateniéndonos a ciertos estudios como los de A. Tovar, la lengua celta fue hablada en las actuales provincias de Soria, Burgos, Logroño, Guadalajara, parte occidental de Teruel y Zaragoza y sur de Navarra. Para Tovar sería un tipo de celta «Hallstático» perteneciente a las invasiones celtas más antiguas: «Corresponden a las invasiones célticas más antiguas, grosso modo, coetáneas de las primeras en Gran Bretaña. No obstante, no existen indicios de su relación con el goidélico insular, y lo más verosímil es aceptar esta lengua como perteneciente a los celtas hallstáticos entrados en la Península hacia los siglos VII VI" ${ }^{1}$. La presencia de estos celtas hispanos por los restos encontrados podemos considerar que tuvieron su núcleo principal de asentamientos en las cuencas del Ebro y del Duero, pese a que sus asentamientos llegarán a amplias regiones de la actual Portugal, extendiéndose hacia el sur por la zona de Extremadura, Madrid y Guadalajara.

1 Tovar, A.: Enciclopedia Lingüistica Hispánica l, CSIC (Madrid), 1960, p. 112. 
«Villastar, Monreal de Ariza, Luzaga, Huete, algún punto no precisado de Castilla la nueva situado entre Cuenca y Madrid, Numancia, Peñalba de Castro, Langa de Duero y Sasamón son las localidades que han dado varias inscripciones notables, en clave celtibérico» ${ }^{2}$.

Para Tovar existieron varias invasiones celtas en la Península. La topografía en -briga podría pertenecer a la primera (hallstática), la segunda invasión, posiblemente gala, nos legaría los topónimos en -dunum. Palabras como camisa, cabaña, cerveza, legua, abedúl, alondra, salmon, carro, carpintero, brio y vasallo son algunas palabras celtas que aparecen en español. Muchas palabras celtas fueron aprendidas también por los romanos e incorporadas al latín... carpentarius (carpintero), betulla (abedúl), carrus (carro). pese a que Antonio Tovar no encuentra relación entre el celta hispano y el goidélico irlandés, Julio Caro Baroja sí encuentra ciertas relaciones. Por ejemplo nos señala el pueblo vasco de nombre celta Deva, y el río Deva que pasa por esa misma población, y su posible relación con el río escocés Dee, que pasa por la ciudad de Aberdeen. Deva y Dee presumiblemente tienen un origen común: el nombre de la diosa Dive guarda relación con la divinización de las aguas fluviales y de las fuentes, podemos considerar que el paso de esta palabra a Escocia pudo tener lugar a través de Irlanda, puesto que la lengua y la cultura gaclica se desarrollaron tanto en Irlanda como en Escocia. Otro río importante español y con una posible relación lingúística es el Duero - Durius o Duria-. En gaélico tenemos la palabra dur-lus que significa agua. Al referirse a Irlanda Ptolomeo cita un río llamado Dur.

La palabra trebo (aldea), aparece en España en el nombre de Contrebia, nombre dado a las ciudades celtrberas, también en el condado inglés de Lancaster, en el noroeste de Inglaterra próximo a Escocia, tenemos un pueblo citado por Livio bajo el nombre de Contrubii ${ }^{3}$. Si ojeamos un diccionario de Irlandés moderno podemos ver la palabra treibh, que significa actualmente tribu. La palabra celta bebros (castor) aparece en España en el nombre gentilicio de bebrices y beribraces en gaelico tenemos beithioch y beathach que significan animal o bestia. Silo Itálico nos menciona un pueblo que habitaba en los valles del Pirineo y que tenía un rey conocido bajo el nombre de bebryx (castor) ${ }^{4}$. En Irlanda O, S'ulleabhain recoge en 1492, en su obra Handbook of Irish Folklo$r e$, un relato según el cual existió un rey nutria ${ }^{5}$.

2 Carro Baroja, J.: Historia de Espatia (Ramón Menéndez Pidal), I (***), Espasa Calpe, 1976, p. 761.

3 Caro Baroja, J.: Los pueblos de España (I), Istmo (Madrid), 1975, p. 93.

4 Ver Caro Baroja, p. 92.

5 S'UuleabhaIN, O.: A Handbook of Irish Folklore, Co. of Ireland Ltd. (Wexford), 1942, p. 590. 
Existe un curioso paralelismo entre el primitivo celta goidélico -del que evolucionaron posteriormente el gaélico irlandés, el gaélico escocés y el Manx (lengua gaélica de la isla de Man)-y el primitivo celta britano lengua originaria del galés, córnico y bretón, con las dos ramas itálicas: latón y osco-umbro. El goidélico y el latín retienen el sonido $/ \mathrm{k} /$, mientras que el britano y el osco-umbro lo convierten en $/ \mathrm{p} /$. Por esta razón se ha pensado que pudo existir una primitiva lengua italo-celta, que más tarde se dividirfa en dos ramas bien diferenciadas el itálico y el celta común. Las diferencias entre el celta $/ \mathrm{k} /$ y el celta $/ p /$ son claramente perceptibles en el vocabulario de las lenguas celtas goidélicas y britanas:

\begin{tabular}{lllll} 
Español & Irlandés & Galés & Córnico & Bretón \\
\hline hijo & mac & map & map & mab \\
cabeza & cenn & pen & pen & penn \\
cinco & cúig & pump, pum & pymp & pemp
\end{tabular}

La mayoria de las inscripciones del celta hispano se encuentran escritas con el alfabeto ibero. Sabemos que la cultura celta era oral y que los primeros manuscritos que poseemos tanto en celta $/ K /$ como en celta $/ P /$ se escribieron en las Islas Británicas usando el alfabeto latino y no antes del s. VI o VII A. D. Por lo tanto las inscripciones hispánicas son bastante más antiguas, pese a que todavía no tengamos una traducción fidedigna de ellas. Debemos de señalar que el alfabeto ibero no tiene el signo $P$, signo de escritura que pese a la evolución anteriormente señalada de los sonidos $/ \mathrm{K} /$ a sonidos $/ \mathrm{P} /$ en las lenguas celtas, también aparece en muchas palabras del celta $/ K /$ que no siguen la evolución anteriormente indicada. A continuación podemos ver los signos ibéricos usados en estas inscripciones celtas ${ }^{6}$ :

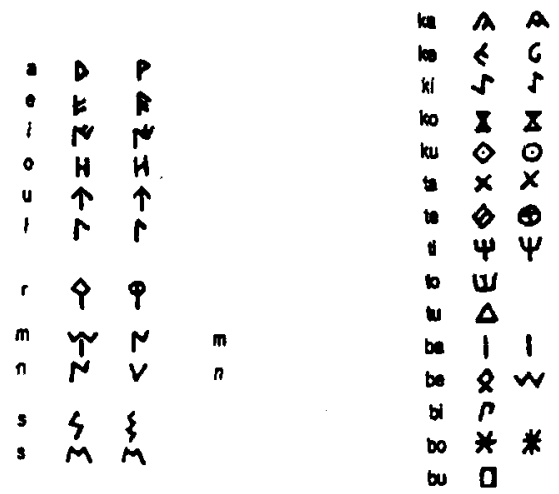

6 Según versión de Francisco Marco en Los celtas en el valle medio del Ebro, Caja de Ahorros de la Inmaculada Aragón, 1989, p. 103. 
Posiblemente la inscripción más significativa de la lengua celta en España es la que aparece en el «Bronce de Luzaga» encontrado en Guadalajara, con ocho líneas escritas y un total de 123 signos. Estos signos o letras son iberos como es habitual en las inscripciones de la Península que para escribir la lengua ibera o celta usan los signos iberos. Los estudios realizados sobre el Bronce de Luzaga nos permiten conocer las palabras pero por desgracia no su significado, la inscripción y su transcripción en signos latinos realizada por Hubner y Gómez Moreno es como sigue:

\section{Bronce de Luzaga (Guadalajara)}

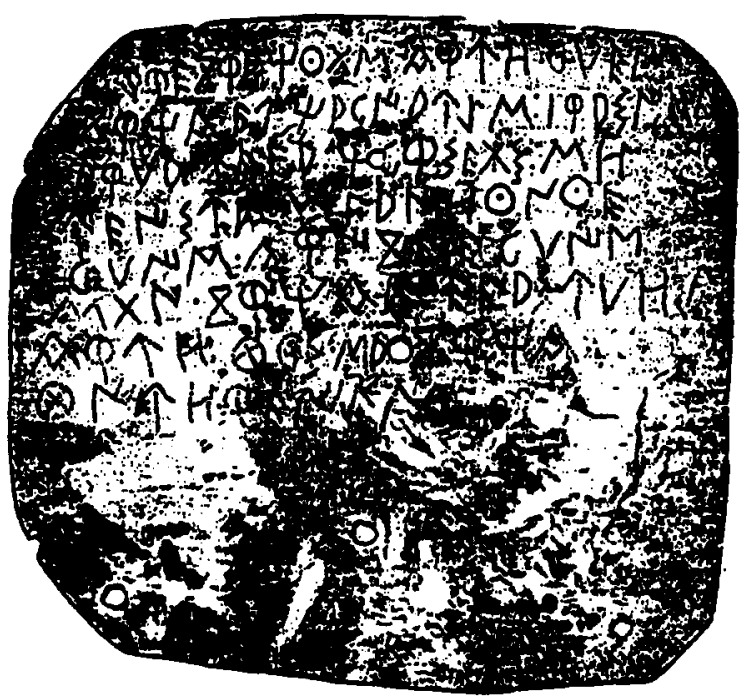

1) AREC(G)ORATICUBOS CARUO CEMEI.

2) C(G)ORTICA LUTIACEI AUGIS BARASIOCA.

3) ERMA UELA TICERSEBOS SO.

4) UEISUI BELAIOCUNCUE.

5) CEMIS CARIGOCUE CEMIS.

6) STAN C(G)ORTICAN ELASUMON.

7) CARUO TUGIS SABA C(G)ORTICA.

8) TUIUOREIGIS.

Como hemos dicho anteriormente los estudios realizados hasta el momento no han podido llegar a su traducción. Lo que sí nos llama la atención al 
leer estas inscripciones es comprobar la gran cantidad de palabras que según la transcripción a signos latinos llevan sonido / $\mathrm{k}$ : ARECORATICUROS, CARUO, CEMEI; CORTICA, BARASIOCA, TICERSEBOS; BELAIOCUNQUE; CEMIS CARIOCUE CEMIS; CORTICAN; CARUO, CORTICA. Ya hemos hablado de las diferencias básicas en las lenguas celtas con sonido $/ \mathrm{k} / \mathrm{o}$ $/ \mathrm{Qu} / \mathrm{y}$ las lenguas celtas con sonidos $/ \mathrm{P} /$ por lo que no vamos a incidir en el tema. La posibilidad de que el celta hispano pertenezca al celta $/ \mathrm{K} /$ y no al $/ \mathrm{P} / \mathrm{pa}$ rece evidente en un principio, no obstante debemos de señalar que la letra y el sonido /p/ sí existe en goidélico, y no en el alfabeto ibero. En la inscripción celtibérica de este bronce podemos ver que las palabras de las 8 líneas tienen gran similitud con palabras del antiguo irlandés (goidélico), ofreciendo bastante sentido a la construcción de la frase, sorpendentemente como veremos más abajo se ajustan con cierta facilidad al celta goidélico:

Para empezar debemos de indicar, quizás lo más importante, que en antiguo irlandés (goidélico), la construcción de la frase es completamente diferente a lo que conocemos en el mundo clásico, y de aquí la gran dificultad para todos aquellos interesados en una posible traducción del celta hispano. Las palabras en antiguo irlandés tal y como aparecen escritas no son muchas veces en realidad palabras, sino conjunto de palabras. Para mejor comprensión veamos algún texto primitivo escrito en antiguo irlandés, lengua que como sabemos pertenece al celta $/ \mathrm{K} /$ :

Tomemos por ejemplo un fragmento del Ciclo del Ulster, escrito en el s. IX A. D. y que aparece en el Leabhar Laigen (Libro de Leinster, recopilado en el s. XII, relato: «De cómo Cuchulainn llegó a Emain Macha, líneas 48554901). Se refiere a las hazañas del héroe mítico Cuchulainn en su niñez: La madre de Cuchulainn no le deja ir a la capital del reino Emain Macha: «Ní regae,» ol a máthair, («No irás, dice su madre,»). A continuación la frase es imposible de traducir a menos que dividamos las palabras en otras palabras: «condit roib coímthecht di ánrothaib Ulad». «Rochían limsa anad fri sodain,» ol Cú Chulainn»

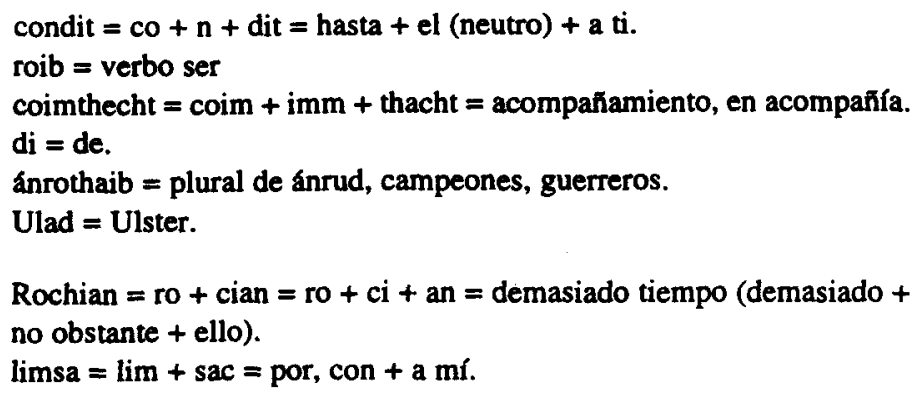

Rochian $=$ ro + cian $=$ ro $+\mathrm{ci}+$ an $=$ demasiado tiempo $($ demasiado + no obstante + ello).

limsa $=\lim +\mathrm{sac}=$ por, con $+\mathrm{a}$ mí. 


$$
\begin{aligned}
& \text { anad = verbo, esperar. } \\
& \text { fri }=\text { por. } \\
& \text { sodain = ese tiempo. } \\
& \text { ol = dice. } \\
& \text { Cú = perro. } \\
& \text { Chulainn = de Culan. }
\end{aligned}
$$

Traducción = Hasta que tu (a ti) seas en compañía de guerreros del Ulster. Demasiado tiempo para mí (con a mí) esperar durante ese tiempo. Dice $\mathrm{Cu}$ + Chulainn (Perro de Culan).

Siguiendo este sistema de traducción las palabras que aparecen en el bronce de Luzaga se pueden descomponer en otras.

Y si realmente el celta hispano es celta /Qu/ pudo que haber sido escrito siguiendo las mismas pautas que el antiguo irlandés. La primera línea:

1) AREC(G)ORATICUBOS CARUO CEMEI se puede descomponer en las siguientes palabras:

1) ARE CORA TI CUBOS CARUO CEMEI.

Vamos ahora a estudiar las palabras por separado, teniendo presente, cuando existan varios o más significados en una palabra, su relación con el resto de las palabra en el texto.

\section{1) ARE-CORA-TI-CUBOS}

ARE (antiguo Irlandés ARE/AIRE) = Acto de hacer una valla, pared o presa.

CORA (antiguo Irlandés CORA) $=$ Muro de piedra, pared, valla . Tl (antiguo Irlandés TI) = Línea, círculo, espacio cerrado por líneas. CUBOS (antiguo Irlandés CUBUS) = Arbol (Irlandés modemo crann). Es sabido que los celtas adoraban en sus creencias religiosas a ciertos árboles.

CARUO (antiguo Irlandés CARA, palabra relacionada con la palabra latina CARO = Borde, lado, amigo. También en ant. irlandés CARAID, verbo débil = amar

CEMEI (antiguo irlandés CIMAS) = Límite de territorio. La forma CEMEI puede ser un genitivo similar en su construcción a la primera declinación de los nombres masculinos gaélicos. Ejemplo BAD (barco), nominativo sing. BAD, genitivo sing. BEID.

También tenemos en antiguo Irlandés C $(\mathrm{H})$ ENEIL $=$ RAZA, TRIBU. La $\mathrm{H}$ que aparece en $\mathrm{A}$. Ir. antes de la $\mathrm{C}$ pudiera ser el conocido fenómeno de aspiración en la lengua gaélica. Tanto en $A$. Ir. como irlandés moderno, nos puede indicar que la palabra anterior rige genitivo por lo que CENEL/CENEIL pasaria a convertirse en 
CHENEL/CHENEIL $=$ De la tribu. No obstante esta segunda posibilidad parece más remota.

Por otro lado también tenemos la construcción CE-MEI en la que CE significa en irlandés moderno «tierra» o «leche» y MEI (en gaélico MISE) pronombre reflexivo «El mismo».

Referente a la Palabra goidélica CORA el diccionario de Antiguo Irlandés de la Royal Irish Academy (1983), nos dice lo siguiente:

«(a) stone fence, palisade, wall, row of serried rank ( $\alpha$ The making of a c. round land (without objection) was evidence of title*, Plummer Ms notes)» p. 151.

«(a) valla de piedra, empalizada, muro, fila o en filas apretadas ( $\ll$ La construcción de una c. (cora = valla) de tierra redonda (sin objección) era evidencia de tútulo», Plummer MS citas)».

Después de todo lo anterior la traducción de la primera línea del bronce de Luzaga podría aproximarse en mayor o menor medida a la siguiente traducción:

«HACER UNA VALLA EN UN ESPACIO CIRCULAR CERRADO (POR LINEAS) UN ARBOL (DE) AMOR (DE) LA TIERRA (TRIBU) MISMA».

O también:

«HACER UNA VALLA EN EL ESPACIO CIRCULAR CERRADO (POR LINEAS) DEL ARBOL AL LADO DEL LIMITE DEL TERRITORIO»

La línea 2) CORTICA LUTIACEI AUGIS BARASIOCA nos ofrece la siguiente relación con respecto al celta irlandés primitivo:

CORTICA (Antiguo irlandés CORTIGE) = dureza, inflexibiliad. LUTIACEI (antiguo irlandés LUTAINGEN, LUTU) = Dedo pequeño. AUGIS (antiguo irlandés AUGA/UCCU) $=$ Deseo, seleccion.

BAR-AS-IOCA (antiguo irlandés BAR) = Líder, hombre sabio, adivino. AS (antiguo irlandes AS) = Acción de incrementar, acción de aumentar. IOCA (antiguo irlandés IOGA/FIOGAR) = Figura, tipo, símbolo.

Referente a LUTIACEI debemos de tener presente la importancia del dedo pequeño en las leyes de los primitivos irlandeses, sabemos que LUTAIN- 
GEN/LUTU, el dedo pequeño en antiguo irlandés, expresaba una unidad de medir: «lutu laime fir domidighthur dia tigut «a man's little finger measures it in thickness» («el dedo pequeño de un hombre mide en grosor», Diccionario de Antiguo Irlandés, Royal Irish Academy, 1983, p. 446). La traducción más próxima puede ser la siguiente:

\section{«LA DUREZA DEL DEDO PEQUEÑO (DE) UN HOMBRE SABIO HACE AUMENTAR EL SIMBOLO»}

La línea 3 del bronce, ERMA UELA TICERSEBOS SO, la podemos relacionar de la siguiente manera:

ERMA (antiguo irlandés ERMA) = Curso, movimiento

UELA (antiguo irlandés BEL/BÉLAIB) = Labios, boca. en antiguo irlandés palabras como UEUSS también aparecen como BEUS en otros textos (ver diccionario Royal Irish Academy, p. 625), por esta razón podemos pensar en la relación UELA/BELA.

TI-CERSEBOS, aquí nos encontramos con una forma parecida a la que aparece en la línea 1 (-TI-CUBOS):

TI (antiguo irlandés TI) = Línea, círculo, espacio cerrado entre líneas. CERSEBOS (¿ARBOL?) $=$ En antiguo irlandés tenemos CEIRT = Manzano y también nombre de la letra $Q$ oghámica.

SO (antiguo irlandés SO) $=$ Demostrativo éste-a.

La traducción de esta línea 3 que podemos ofrecer, más confusa y menos fiable que las anteriores, podría ser:

*UN MOVIMIENTO DE LABIOS EN EL CIRCULO DEL ARBOL ESTE».

La línea 4: «UEISUI BELAIOCUNCUE» nos ofrece las siguientes correlaciones:

UEI-SUI (antiguo Irlandés UEL/BEI, verbo BERID)= llevar.

SUI Antiguo irlandes SUI= Hombre sabio, sabio.

BELA-I-OCUNCUE (antiguo irlándés BELA)= Movimiento de labios, labios. es la misma palabra que aparece en la línea 3, escrita UE$L A$, esto nos muestra la alternancia entre U-/E que también aparece en antiguo irlandés.

I-(antiguo irlandés $\mathrm{I})=$ Pronombre demostrativo éste a-o.

OCU (antiguo irlandés $\mathrm{OC}$ ) $=$ Preposición, en .

$\mathrm{N}$ (antiguo irlandés $-\mathrm{N}-$, articulo, nominativo, neutro) $=$ ello.

CUE (antiguo irlandés CUE/CUA) = Cavidad, hueco. 
Después de lo anterior la línea 4 no puede quedar traducida de la siguiente manera:

\section{«PORTA UN HOMBRE SABIO EL MOVIMIENTO DE LABIOS ESTE EN $i .$. ? CAVIDAD».}

Veámos a continuación la línea 5: CEMIS CARIGOCUE CEMIS.

Como vimos anteriormente en el estudio de la línea 1 CEMEI (antiguo irlandés CIMAS) = Límite de territorio, la forma CEMEI puede ser un genitivo similar en su construcción a la primera declinación de los nombres masculinos gaélicos. Ejemplo BAD (barco), nominativo sing. BAD, genitivo sing. BEID.

También hemos visto, aunque menos probable, que CEMEI (CHENEIL) podría significar en antiguo irlandés: «tribu»,»raza».

Por último también tenemos otra forma CEMIS en antiguo irlandés. En la palabra CEMIS podemos ver otra relación con la palabra que aparece en la línea 1: CEMEI, y que existe en el antiguo irlandés en el compuesto CE + MEI $=$ «En este lado de Mei». MIS según el diccionario de An. Irl. de la Royal Irish Academy es una forma de genitivo $e$ indica nombre de lugar.

CAR-IGOCUE CAR=UN GIRO, UNA VUELTA (GAELICO) (antiguo irlandés CAR= Amado, querido), CARA (antiguo lrlandés)= Lado, IGOCUE (antiguo irlandés IGA/IDU)= Ley (IGHAN= leyes), CUE (antiguo irlandés CUE/CUA)= Agujero, cavidad, espacio vacío. CEMIS ya la hemos estudiado con antelación.

La línea 6 nos ofrece el texto siguiente. «STAN C(G)ORTICAN ELASUMON»

STAN (antiguo irlandés STAN) $=$ estaño.

C(G)OR-TI-CAN (antiguo irlandés COR/CORA, nombre verbal de co-feird $)=$ Acto de emplazar, asentar.

TI palabra ya estudiada en las líneas 1 y 3= Línea, círculo, espacio cerrado por líneas.

CAN (antiguo irlandes CAN/CIAN)= largo tiempo, espacio de tiempo, distante.

Forma escrita ELASUMON. Se puede dividir en ELA-SUMON: ELA en antiguo irlandés EOL (genitivo singular EOLA) = Conocimiento, sabiduría, habilidad.

SUMON en antiguo irlandés tenemos la forma enfática SUM que también aparece esccrita como SOM y SAM. Tenemos también la forma SOMMAE 
(quizá también SUMMAE siguiendo el modelo de SUM = Riqueza, valores; y también tenemos SONNAN (¿SUNNAN?)= Tierra próspera.

La posible traducción sería la siguiente: «ESTAÑO ASENTADO EN UN ESPACIO CIRCULAR CERRADO POR LINEAS LARGO TIEMPO CONOCIDO COMO (CONOCINIENTO DE) TIERRA PROSPERA (VALIOSA)».

Línea 7: «CARUO TUGIS SABA C(G)ORTICA».

La forma CARUO aparece en la línea 1 y ya ha sido estudiada = Borde, lado. amigo.

TUGIS antiguo irlandés TUIGE (nombre verbal de TUIGITHIR) $=$ Acto de cubrir o proteger.

SABA en antiguo irlandés SABAIL = Acto de salvar, proteger. Quizá préstamo del latín SALVARE.

La forma C(G)ORTICA la hemos estudiado en la primera línea = Dureza, inflexibilidad. No obstante la forma $C(G) O R T I C A$ nos recuerda la forma en la línea 1 ya estudiada «CORA-TI-» $=$ CORA = Muro de piedra, pared; TI = valla-línea, círculo, espacio cerrado por líneas.

La traducción de esta línea 7 podría aproximarse a lo siguiente:

«LADO (BORDE) QUE CUBRIR PARA SER PROTEGIDO (CON) DUREZA».

O también:

«LADO QUE CUBRIR PARA SER PROTEGIDO CON UNA VALLA»

Por último la línea 8 nos ofrece una sola forma: «TUIUOREIGIS».

Forma que podemos dividir en las siguientes palabras del antiguo irlandés TUI-UO-REGIS, vamos ahora a analizarlas:

TUI nos recuerda la palabra TUIGIS que acabamos de estudiar en la línea $7=$ Acto de cubrir o proteger. También tenemos en antiguo irlandés la palabra TUL/TOE $=$ Silencio, silenciosamente, pero parece menos probable.

UO en antiguo irlandés tenemos UR/URA = Con referencia a lugar: verde, freco-a (prado, colina). También $\mathrm{OR}=$ Nombre de la planta del brezo. Por último podemos decir que OR en antiguo irlandés puede significar también «oro» (latín AURUM).

En cuanto a REIGIS nuestro primer pensamiento es asimilarlo a la palabra latina REX, genitivo singular REGIS= Rey, del rey. Pero en antiguo irlandés rey aparece como RI y en genitivo de singular como RIOGH, por lo que si no existe una influencia latina directa tendremos que pensar más bien en la palabra gaélica primitiva REIGLEN= Campo horizontal, prado, ¿yacimiento?

La traducción de esta línea 8 nos puede ofrecer lo siguiente:

PROTEGIENDO (CUBRIENDO) EL VERDE CAMPO HORIZONTAL. o también:

PROTEGIENDO (CUBRIENDO) EL CAMPO (YACIMIENTO) DE ORO. 
Por el contexto de las frases podemos seleccionar las palabras estudiadas anteriormente y tener una traducción aproximada al contenido de la inscripción celtiberica:

1) AREC (G) ORATICUBOS CARUO CEMEI.

2) C (G) ORTICA LUTIACEI AUGIS BARASIOCA.

3) ERMA UELA TICERSEBOS SO.

4) UEISUI BELAIOCUNCUE.

5) CEMIS CARIGOCUE CEMIS.

6) STAN C (G) ORTICAN ELASUMON.

7) CARUO TUGIS SABA C(G)ORTICA.

8) TUIUOREIGIS.

Traducción:

1) «HACER UNA VALLA EN EL ESPACIO CIRCULAR CERRADO (POR LINEAS) CON EL ARBOL AL LADO DEL LIMITE DEL TERRITORIO».

2) «UN MOVIMIENTO DE LABIOS (UN MANDATO) EN EL CIRCULO DE ESTE ARBOL».

3) «LA DUREZA DEL DEDO PEQUENO (DE) UN HOMBRE SABIO HACE AUMENTAR EL SIMBOLO».

4) «LLEVA UN HOMBRE SABIO EL MOVIMIENTO DE LABIOS (EL MANDATO) A ESTA CAVIDAD».

5) «EL LIMITE DEL TERRITORIO EN EL BORDE ¿LEGAL? DE LA CAVIDAD».

6) «EL ESTAÑO ASENTADO EN UN ESPACIO CIRCULAR CERRADO LARGO TIEMPO CONOCIDO COMO TIERRA PROSPERA».

7) «LADO QUE CUBRIR PARA SER PROTEGIDO CON UNA VALLA»

8) «CUBRIENDO EL CAMPO ¿DE ORO?».

Pese a todo lo anterior en esta traducción existen muchas dudas morfosintácticas de difícil, quizá imposible, solución. Una cosa que llama la atención es la ausencia de artículo determinado —en antiguo irlandés aparece como in, int nominativo masculino, ind, in, int nominativo femenino, a $n-,-n-$ nominativo neutro, ... etc». Solamente tenemos un signo - n-en toda la inscripción, aparece en la línea 4 en la forma «BELAIOCU-N-CUE», de la que 
podríamos pensar en la posibilidad de un artículo neutro (ver traducción de la línea 4). Referente al artículo indeterminado sabemos que tanto en antiguo irlandés como en irlandés moderno no existe escrito. La ausencia de artículo delante de un nombre indica la ausencia de artículo o que se sobre entiende el artículo indeterminado, ejemplo: far «hombre», «un hombre»; an far «el hombre».

Podemos objetar con razón que son muchos siglos los que separan es texto del bronce de Luzaga de los textos escritos en antiguo irlandés que comienzan a escribirse en el s. VII A. D. como época más temprana. Pero ante esto podemos decir que la lengua gálica tiene la extraordinaria particularidad de haber sufrido variaciones mínimas durante más de mil años. Bástenos ver el texto senalado anteriormente referente a Cuchulainn que aparece en varios manuscritos (Leabhar Laigen y Leabhar na h-uidre entre otros, ambos recopilados en el s. XII), el documento pertenece a la lengua hablada en el s. IX y que debía de ser la misma o con muy escasas variaciones de la hablada a comienzos de la era cristiana. Podemos ver que muchas de las palabras que aparecen en estos textos son exactamente iguales a las usadas en pleno siglo $\mathrm{XX}$ en la lengua gaélica moderna, y otras muchas son muy parecidas. Veámos como ejemplo el texto anteriormente citado de Cucuchulainn: «De Cómo Cuchulainn llegó a Emain Macha, líneas 4855-4901 J. «Ní regae,» ol a máthair, («No irás, dice su madre,»). «condit roib coímthecht di ánrothaib Ulad». «Rochran limsa anad fri sodain,» ol Cú Chulainn».

\author{
ANTIGUO IRLANDES \\ $\mathrm{Ni}=$ no \\ regae $=$ fut. verbo teit: irás. \\ ol= dice, dijo (indeclinable). \\ a= Posesivo: su. \\ mathair $=$ madre. \\ condit (co+n+dit): \\ $\mathrm{Co}=$ que, \\ ¿cómo?. \\ $\mathrm{n} / \mathrm{an}=$ pronombre infijo, 3a \\ persona: 61 . \\ dit $=$ pron. + preposicion: a ti. \\ roib= verbo ser, pasado...
}

\author{
IRLANDÉS MODERNO \\ $\mathrm{N} i=$ no \\ rachaidh $=$ fut. verbo \\ teim: irás. \\ $\mathrm{a}=$ posesivo: su. \\ mathair $=$ madre.
}

$c o=$ quien (antiguo

irlandés co-te).

an= artículo: él, ella.

duit $=$ pro. + preposicion

(do+tú): a ti.

raibh: verbo ser,

pasado (después de

particulas): 
coimthecht (coim+imm+thacht):

coim= acompañamiento, en compañáa.

imm= preposicion: alrededor

thecht= forma aspirada de techt:

acto de ir, acto de venir.

di/de= preposicion: de.

ánrothaib= plural de ánrud:

campeones, guerreros.

Ulad $=$ del Ulster.

coimh= prefijo: con.

im= preposición:

alrededor.

teacht $=$ acto de venir.

de $=$ preposición: de.

ánradhd: campeón,

guerrero (plural

anraidh).

Ulaidh= Ulster; Uladh:

del Ulster.

rochian (rotcitan)

ro cia an

$\mathrm{ro}=$ demasiado.

ro= demasiado.

$\mathrm{ci}=$ no obstante.

cia/ce = quien.

$a n / n=$ Artículo neutro.

an = articulo el, ella.

limsa (la+m+sa):

liom sa (le+me sa):

la= preposicion: con.

le= preposición: con.

$\mathrm{m} / \mathrm{m}=$ pronombre personal: $\mathrm{m}$.

sa= sufijo enfático: mismo.

me $=$ pronombre personal $=$

mi .

sa= sufijo enfático.

anad= verbo: esperar.

fanacht $=$ Verbo:

esperar.

fri $=$ por, con

fri $=$ preposición

letpronombre: con el.

sodain $($ so+dain $)=$ buena

suerte, suceso.

so dain= este destino,

este regalo.

Si sorprendente es la relación entre el antiguo irlandés (goidélico) y el irlandés moderno (gaélico), no menos sorprendente es la relación existente entre el alfabeto ibero primitivo en el que fueron escritos los textos celtas en la Península Ibérica y las runas que usaron celtas y germanos en la Europa central y nórdica a comienzos de la Era Cristiana. Runas que sorpendentemente son iguales a los signos iberos. Veámos en el gráfico que ofrecemos a continuación los signos de escritura iberos y rúnicos para ver con claridad esta relación y sobre la que realizamos un estudio más detallado en mi libro La huella celta en España e Irlanda (capítulo 9: «El posible origen hispano de los oghams pictos y las runas germanas»), ed. Akal (Madrid), 1987: 


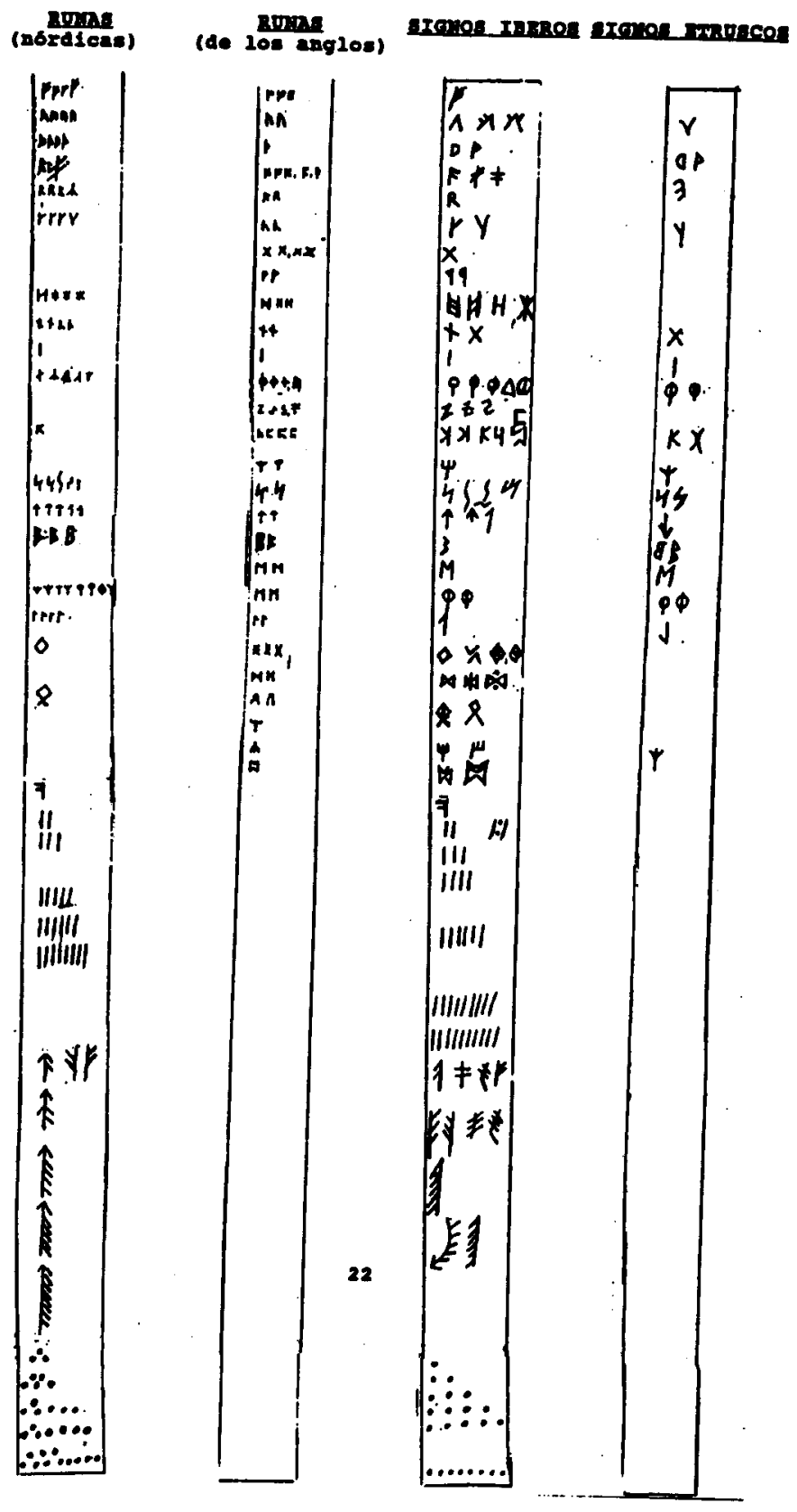




\section{CONCLUSIÓN}

No vamos a entrar en una explicación de la relación entre runas y signos iberos, puesto que escapa a nuestro trabajo basado en un estudio del celta hispano. Podemos terminar diciendo que el método seguido en este estudio del bronce de Luzaga puede ser un camino a seguir en posteriores traducciones. Si no fuera así, nos cabría por lo menos la satisfacción de haber aportado un granito de arena más en este campo de investigación, una visión más dentro de las posibilidades de estudiar estas inscripciones. Nuevas inscripciones celtas siguen apareciendo en España. Tal es el caso del yacimiento celtíbero de Contrebia Belaisca, próximo al yacimiento celta de botorrita, y ambos no muy alejados de la ciudad de Zaragoza, sabemos que fue un enclave celta destruido en el siglo II antes de Cristo, vuelto a edificar y de nuevo destruido en posteriores luchas contra los romanos, se considera de gran valor por los restos celtas encontrados en él entre los que destaca un magnífico bronce escrito en celta que acaba de ser descubierto. Todavía este yacimiento no ha sido desenterrado en su totalidad y los nuevos hallazgos escritos pueden ser de gran importancia. Sin lugar a dudas la labor más sugestiva que tenemos en estos momentos es poder llegar a traducir todas estas inscripciones que permitirían que la morfosintaxis del celta hispano pudiera ser ampliamente estudiada y ser considerada como la lengua celta más antigua de Europa en cuanto a sus restos escritos. 